\title{
10 Gbit/s mode-multiplexed QPSK transmission using MDM-to-MFDM based single coherent receiver for intra- and inter data center networking
}

\author{
Rameez Asif ${ }^{1,2}$ and Mustafa Haithem ${ }^{3}$ \\ ${ }^{1}$ Center for Photonic Systems (CPS), Electrical Engineering Division, Department of \\ Engineering, University of Cambridge, Cambridge (CB3 OFA), UK \\ ${ }^{2}$ Now working with Center for Distributed Computing, Networks and Security, School of \\ Computing, Edinburgh Napier University, Edinburgh (EH10 5DT), UK \\ ${ }^{3}$ School of Engineering, Case Western Reserve University, Cleveland, Ohio (44106), USA
}

\begin{abstract}
Generalized few-mode-fiber (FMF) transmission uses $N$ coherent receivers for mode detection, where $N$ scales with the number of fiber modes. Multiple coherent receivers increase the cost of optical network units (ONUs) in access networks, specifically for intra- and inter data center applications. We have experimentally evaluated a cost effective low-complexity receiver architecture based on mode-division multiplexing (MDM) to mode frequency-division multiplexing (MFDM) conversion for data center connectivity. A single coherent receiver is used to efficiently detect $10 \mathrm{Gbit} / \mathrm{s}$ QPSK Nyquist signals over $2.8 \mathrm{~km}$ 4-LP mode graded index fiber reducing the ONUs complexity to $N / 4$. All the transmitted modes are successfully detected below the forward-error-correction (FEC) limit, i.e. $1 \times 10^{-3}$ BER.
\end{abstract}

Keywords: Optical fiber communication, Space division Multiplexing, Coherent receivers, Networks, Data Centers, Spatial light modulators.

2010 MSC: 78A02, 94A14, 94A13

\footnotetext{
${ }^{1}$ Corresponding Email: mra46@cam.ac.uk
} 


\section{Introduction}

Since the capacity of optical fiber communication systems based on single mode fiber (SMF) is approaching the non-linear Shannon limit, researchers are paying attention to explore new paradigms including advanced coherent modulation formats, multiplexing techniques and specialized fibers [1]. While the use of classical SMF is economical but on the other hand intra-, inter-channel fiber non-linearities (optical Kerr effects) and their inter-play with differential mode group delay (DMDG) still remain the vital performance degrader in high speed conventional coherent systems. Digital signal processing (DSP) based non-linear equalization has been proposed as potential candidate to overcome the physical limitations imposed by the fiber channel. But the computational efforts, in terms of number of complex multipliers and required number of samples to encompass the optical channel memory, need to be simplified. As far as, the required DSP resources are concerned, real-time hardware demonstration of these non-linear equalizers is not yet foreseen in the near future due to the high complexity logic.

[Figure 1 about here.]

Spatial Division Multiplexing (SDM) 2] has been investigated as an alternative approach that has recently being advanced by the research community, albeit being proposed many years ago in the form of multi-mode fiber transmission [3. The use of multi-core fibers (MCFs) and few-mode fibers (FMFs) can substantially increase the transmission capacity per optical fiber beyond the non-linear Shannon limit of SMF systems by offering parallel paths for data communication [4, 5, 6]. The transmission through MCF is considered as a smooth transition from SMF due to the fact that off-the-shelf transceivers and devices can be used for its implementation [7. Whereas, FMF based transmission not only needs specialized multiplexers and demultiplexers [8] but also phase plates or spatial light modulators (SLMs) for generating the respective modes. These spatial modes generally exhibit DMGD and differential modal 
loss/gain. To mitigate these linear impairments, equalization by multiple-input multiple-output (MIMO) DSP is required at receiver. Furthermore, the number of coherent receivers required for efficiently detect the linearly polarized (LP) modes are directly proportional to the number of modes transmitted in the fiber, as shown in Fig. 1(a). In addition to the increase in receiver system complexity, the conventional coherent scheme requires tight skew adjustments among all the received modes after fiber propagation to effectively equalize the received signals by adaptive MIMO DSP algorithms [9, 10. Moreover, the DSP complexity of

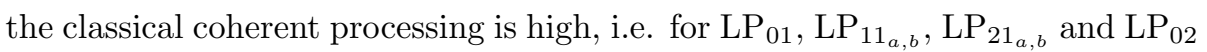
modes we have to implement $2 \times 2,4 \times 4,4 \times 4$, and $2 \times 2$ MIMO blocks, respectively. Collectively, the receiver architecture including DSP is power hungry. This characteristic of FMF based network made it effectively expensive that is impractical for access and data center applications. Most recently, all-optical fiber based mode selective photonic lanterns have been demonstrated for reducing the complexity of MIMO processing [11. The limitation of this device is that they are not flexible in terms of scalability and adaptability. Furthermore, the performance of photonic lantern is immensely dependent on the fabrication process [12]. On the other hand, DSP based mode selective receivers have been successfully demonstrated [13, 14, but the studies are only limited to back-toback system configurations. Most recently, advancements have been made to remove the capacity crunch at the access networking level [15] via coherent receiver implementaion [16, 17] and in data networking architectures [18, through space division multiplexing. However, the complexity of receiver architecture especially for the FMF transmission is still unreasonable for cost-effective implementation of high bandwidth services.

In this paper, for the first time to the best of our knowledge, we have experimentally validated the transmission of 4 -LP modes in $2.8 \mathrm{~km}$ of graded index fiber at the data rate of $10 \mathrm{Gbit} / \mathrm{s}$ by modulating QPSK Nyquist signals and mode selective receiver, as shown in Fig. 1(b). All the LP modes are successfully received via one coherent receiver based on cost effective low-complexity architecture, i.e. MDM to MFDM conversion. The single polarization diversity 
heterodyne receiver with $4 \times 4$ MIMO equalization is employed. The transmission performance is further validated in-terms of bit-error-rate (BER) at diverse wavelengths to emulate the bi-directional traffic, i.e. uplink and downlink. Furthermore, the system performance w.r.t the transmission distance is analytically investigated under practical operating conditions. These results are helpful for the future implementation of SDM-FMF at intra- and inter data center networking level where cost vs. performance in an important parameter.

[Figure 2 about here.]

\section{Experimental Setup}

Fig. 2 depicts the experimental setup of 4-LP mode transmission based on single polarization Nyquist QPSK modulation. A pseudo-random binary sequence (PRBS) of length $2^{31}-1$ is encoded and four delay-decorrelated copies are generated. For laser source, we have used a commercially available multi-channel optical system (ILX Lightwave FOM-7900X) with $1 \mathrm{MHz}$ linewidth. As a result, four $2.5 \mathrm{Gbit} / \mathrm{s}$ (aggregate $10 \mathrm{Gbit} / \mathrm{s}$ ) QPSK Nyquist signals are generated having same wavelength $\lambda$ of $1552.50 \mathrm{~nm}$ (corresponding frequency, $f=192.1$ $\mathrm{THz}$ ), i.e. $\lambda_{1}, \lambda_{2}, \lambda_{3}$ and $\lambda_{4}$ have the same wavelength as $\lambda_{\text {sig. }}$ Moreover, the resultant pre-amplified, decorrelated and filtered optical signals are incident on a liquid-crystal on silicon spatial light modulator (Hamamatsu LCOS-SLM X13138 series) with light utilization efficiency of $\sim 80 \%$, generating the respective $\mathrm{LP}_{01}, \mathrm{LP}_{11 a}, \mathrm{LP}_{11 b}$ and $\mathrm{LP}_{21}$ modes. These signals are multiplexed together and launched in the 4-LP mode graded index fiber. Due to the limitations of resources in the laboratory we have only incorporated $2.8 \mathrm{~km}$ of fiber in the experiment with cladding diameter of $125 \pm 0.7 \mu \mathrm{m}$. The dispersion parameters for $\mathrm{LP}_{01}, \mathrm{LP}_{11}$ and $\mathrm{LP}_{21}$ modes are $18.4 \mathrm{ps} /(\mathrm{nm} . \mathrm{km}), 18.7 \mathrm{ps} /(\mathrm{nm} . \mathrm{km})$ and 18.0 ps/(nm.km), respectively. The differential group delay (DGD) between all the modes is $\pm 0.4 \mathrm{ps} / \mathrm{m}$. In this experiment, the local oscillator (LO) module comprises of multi-channel laser source and LCOS-SLM. The central frequency of 
the four LOs are: $2.5 \mathrm{GHz}, 4.75 \mathrm{GHz}, 7 \mathrm{GHz}$ and $9.25 \mathrm{GHz}$ higher than the frequency for transmitted $\mathrm{LP}_{01}, \mathrm{LP}_{11 a}, \mathrm{LP}_{11 b}$ and $\mathrm{LP}_{21}$ modes repetitively. Each individual pre-amplified and filtered LO signals are then converted into $\mathrm{LP}_{01}$, $\mathrm{LP}_{11 a}, \mathrm{LP}_{11 b}$ and $\mathrm{LP}_{21}$ modes using LCOS-SLM. These signals are connected to the coherent receiver front end with the help of a fiber coupler supporting 4-LP modes. The $20 \mathrm{GHz}$ photo-diodes are used for coherent detection, further assisted by digital storage oscilloscope with sampling rate of $100 \mathrm{GS} / \mathrm{s}$ and analog bandwidth of $50 \mathrm{GHz}$. After converting the MDM signal to the MFDM signal using mode-selective coherent detection technique, analogue-todigital converters (ADCs) digitize the received signal for MIMO processing. The power spectral density of the received signal after transmission is depicted in

Fig. 2. The detailed receiver and signal processing architecture is explained in Appendix A. To get the efficient transmission performance, the $\frac{T}{2}$ spaced finite impulse response (FIR) filters for adaptive MIMO equalization are optimized to be 31-taps. The realistic analytical model for emulating the longer transmission distances is also discussed in the next section.

[Figure 3 about here.]

\section{Results and Discussion}

We first optimize the insertion losses to improve the power budget of the transmission link to get the efficient performance of MDM to MFDM conversion. We have evaluated 4-LP mode $10 \mathrm{Gbit} / \mathrm{s}$ Nyquist QPSK signals at $1552.50 \mathrm{~nm}$. All the received data tributaries are quantified w.r.t received power and BER. The results of 4-LP mode transmission over $2.8 \mathrm{~km}$ of FMF are depicted as in Fig. 3. The back-to-back receiver sensitivity at BER of $10^{-3}$ is $-24.6 \mathrm{dBm}$. After detecting the signals via MDM to MFDM based coherent receiver the power penalties are measured as: $1.2 \mathrm{~dB}, 2.02 \mathrm{~dB}, 2.35 \mathrm{~dB}$ and $3.25 \mathrm{~dB}$ for $\mathrm{LP}_{01}, \mathrm{LP}_{11 a}, \mathrm{LP}_{11 b}$ and $\mathrm{LP}_{21}$ modes, respectively. The variance in the power penalty among different modes are mainly due to the scattering and coupling losses between LCOS-SLM and FMF. For this experiment, we have considered 
back-to-back measurements without optical distribution network (ODN) that includes fiber and LCOS-SLM. The measured power penalties can be reduced by opting efficient coupling schemes between the above mentioned interfaces. In order to emulate the bi-directional traffic scenario in the context of intra-, inter data center networking or next-generation passive optical networks (NGPONs), we experimentally evaluated the receiver scheme at various wavelengths mainly in the C-band. While, the same methodology is adopted to assign the LO wavelengths as described previously. The results, at BER of $10^{-3}$, are plotted in Fig. 4. It can be seen that all the LP-modes can be successfully detected at different wavelengths. The maximum power penalty is recorded as $3.5 \mathrm{~dB}$ for $\mathrm{LP}_{21}$ mode w.r.t back-to-back transmission.

[Figure 4 about here.]

Furthermore, to evaluate the feasibility of MDM to MFDM based receiver over access networking distances, using analytical techniques, we simulated the transmission set-up under practical operating conditions. The simulated results for $\mathrm{LP}_{01}$ mode are depicted in Fig. 5(a). We kept the transmission parameters constant, including laser linewidth, input power, wavelength assignment, in-order to have a relative comparison with the experiment. It can be seen that the performance of the system with the MDM to MFDM based receiver is efficient for distances up-to $33 \mathrm{~km}$ transmission length. This limitation is mainly due to OSNR degradation and filter-taps. While, the performance can further be enhanced by opting $20 \%$ overhead for FEC processing. The receiver bandwidth required to transmit $n-\mathrm{LP}$ modes (where $\mathrm{n}=1,2,3, \ldots, 15$ ) is also analysed. The results are as shown in Fig. 5(b). It is clear from the results that high bandwidth receivers $(>60 \mathrm{GHz})$ can be used for detecting n-LP modes for single polarization transmission that will give an aggregate data rate of 37.5 Gbit/s $/ \lambda(15$-LP modes $\times 2.5 \mathrm{Gbit} / \mathrm{s})$. Whereas adding another coherent receiver will allow the user to detect dual-polarization signals that will increase the transmission capacity of the network by a factor of $\times 2$. By considering these results, we would like to mention that the use of such kind of receiver architec- 
ture is ideal for low baud-rate signals and number of modes, so that they can be accommodated with the fixed bandwidth of the receiver. Despite of the fact that high bandwidth commercial coherent receivers are available but either they are expensive or they need complex ADCs to process the data. Hence making them inappropriate to be used in access networks and data center applications where cost/economy is the primary concern. These feasibility results are helpful for the implementation of high-spectrally efficient few-mode fiber networks as well as for dual-polarization transmissions.

[Figure 5 about here.]

\section{Conclusion}

We propose and experimentally demonstrate a low-complexity receiver architecture for transmitting 4-LP modes for short range fiber links and data center applications. We have successfully transmitted single-polarization 4-LP modes over $2.8 \mathrm{~km}$ of graded index fiber at the data rate of $10 \mathrm{Gbit} / \mathrm{s}$ by modulating QPSK Nyquist signals and detecting them with single coherent receiver. Furthermore, the receiver architecture is evaluated at different wavelengths to emulate the bi-directional traffic. While extended simulation studies have been performed to quantify the receiver performance over longer distances and receiver bandwidth w.r.t transmittable LP modes. To conclude, SDM based lowcost spectrally efficient coherent architectures can be deployed to remove the capacity crunch at access or date center networking level and to provide nextgeneration services to the subscribers.

\section{Appendix A: Mode-Selective Coherent Detection Technique}

The proposed receiver is based on the conversion of mode division multiplexed (MDM) signal into mode frequency division multiplexed (MFDM) signal via local oscillator ( $\mathrm{LO}$ ) that comprises of a laser bank. The MDM signal, $\mathrm{E}_{\text {sig }}$ and MFDM LO, $\mathrm{E}_{L O}$, can mathematically be expressed as follows in Eq. 1 and Eq. 2 . 


$$
\begin{gathered}
E_{s i g} \approx \sum_{m=1}^{M} A_{m} E_{m} e^{j 2 \pi f_{s i g_{m}} t} \\
E_{L O} \approx \sum_{n=1}^{N} A_{n} e^{j 2 \pi f_{L O_{n}} t}
\end{gathered}
$$

Where, $\mathrm{E}_{m}$ is the complex field amplitude of the $\mathrm{m}^{\text {th }}$ modulated signal and $j$ is the imaginary part of the complex envelope. Furthermore, $\mathrm{A}_{m}$ and $\mathrm{A}_{n}$, respectively, are $\mathrm{m}^{t h}$ and $\mathrm{n}^{\text {th }}$ propagation modes of MDM and MFDM's LO. In out experimental demonstrations, $\mathrm{M}$ and $\mathrm{N}$ are equal to 4 . The received electrical signal, I, output from the photo-detectors in the coherent receiver, as given by the received MDM signal and MFDM LO, can mathematically expressed as in Eq. 3 .

$$
I \approx \sum_{m \geq 1}^{M} \sum_{n \geq 1}^{N} A_{m} A_{n} E_{m} e^{j 2 \pi\left(f_{s i g_{m}}-f_{L O_{n}}\right) t}
$$

The MDM signal comprising the LP modes has orthogonality property as per Eq. 4.

$$
A_{m} A_{n}=\delta_{m n}=\{1,(m=n) 0,(m \neq n)
$$

Where, $\delta_{m n}$ is the Kronecker delta that narrates piecewise function of the discrete modes having variables $\mathrm{M}$ and N. By considering Eq. 3 and Eq. 4. coherent beat signals are selectively detected among the same propagation modes of the MDM signal and the MFDM LO as shown in Fig. 11 Therefore, the MDM signal can be converted into an FDM signal in the coherent detection scheme that can be expressed as in Eq. 5.

$$
I \approx \sum_{m \geq 1}^{N} E_{m} e^{j 2 \pi\left(f_{s i g_{m}}-f_{L O_{m}}\right) t}
$$

After converting the MDM signal to the MFDM signal in the coherent receiver using the proposed mode-selective coherent detection technique, ADCs digitise the input signal. Then, the respective DSP module down-converts the 
frequency-shifted signals, i.e. 4 signals corresponding to each individual LP mode, to baseband signals. Each baseband signal is input to a low-pass filter (LPF) to remove out-of-band FDM signals. The DSP also applies chromatic dispersion compensation with frequency domain equalisation [9, adaptive MIMO equalisation with a finite impulse response (FIR) filter and carrier phase recovery [10].

\section{Acknowledgment}

The authors would like to thank Prof. Toshio Morioka, Prof. Seb Savory and Assoc. Prof Darko Zibar for their valuable discussions and guidance.

\section{Author Contributions Statement}

R.A conceived the work and carried out the experimental work. R.A and M.H implemented the digital signal processing for the coherent receiver. M.H performed further analytical calculations. R.A and M.H conducted the whole project. Both authors discussed the results and contributed to the final manuscript.

\section{References}

[1] G. Li, "Recent advances in coherent optical communication," Adv. Opt. Photon, vol. 1, no 2, pp. 279-307, February 2009.

[2] D.J. Richardson, M.J. Fini and L.E. Nelson, "Space-division multiplexing in optical fibers," Nature Photonics, vol. 7, pp. 354-362, April 2013.

[3] H. Masaaki, "Future of Transmission Fiber," IEEE Photon. J. , vol. 3, no. 2, pp. 316-319, April 2011.

[4] S. Ark, K. Ho and J.M. Kahn, "Optical network scaling: roles of spectral and spatial aggregation," Opt. Express , vol. 22, no. 24, pp. 29868-29887, November 2014. 
[5] T. Mizuno, H. Takara, A. Sano and Y. Miyamoto, "Dense space division multiplexed transmission systems using multi-core and multi-mode fiber," J. Lightwave Technol, vol. 34, no. 2, pp. 582-592, September 2015.

[6] R. Asif, "Advanced and flexible multi-carrier receiver architecture for highcount multi-core fiber based space division multiplexed applications," Sci. Rep. 6, 27645, June 2016.

[7] H. Hu, R. Asif, F. Ye, S. Gross, M. Withford, T. Morioka and L. Oxenlowe, "Bidirectional 120 Gbps SDM-WDM-PON with colourless ONU using 10 Gbps optical components without DSP2," Optical Fiber Communication Conference (OFC), Anaheim (California), paper MC3.1, March 2016.

[8] R. Asif, H. Hu, P. Mitchell, J. Macdonald, F. Da Ros, N. Psaila, F. Ye, T. Morioka and L. Oxenlowe, "Experimental demonstration of 6-mode division multiplexed NG-PON2: cost effective $40 \mathrm{Gbit} / \mathrm{s} / \mathrm{spatial}$-mode access based on 3D laser inscribed photonic lanterns," European Conference on Optical Communications (ECOC), Valencia (Spain), paper Tu.1.5.1, September 2015.

[9] N. Fontaine, R. Ryf, H. Chen, A. Benitez, B. Guan, R. Scott, B. Ercan, S.J.B Yoo, L. Gruner-Nielsen, Y. Sun, R. Lingle, E. Antonio-Lopez and R. Amezcua-Correa, "30×30 MIMO transmission over 15 spatial modes," Optical Fiber Communication Conference, post deadline papers, paper Th5C.1, Los Angeles, United States 2015.

[10] R. Asif, F. Ye and T. Morioka, "Equalizer complexity for 6-LP mode 112 Gbit/s m-ary DP-QAM space division multiplexed transmission in strongly coupled Few-Mode-Fibers," European Conference on Networks and Communications (EuCNC), pp. 317-320, Paris France, 2015.

[11] S. Leon-Saval, N. Fontaine, J. Salazar-Gil, B. Ercan, R. Ryf and J. BlandHawthorn, "Mode-selective photonic lanterns for space-division multiplexing," Opt. Express, vol. 22, no. 1, pp. 1036-1044. January 2014. 
[12] R. R. Thomson, T. A. Birks, S. G. Leon-Saval, A. K. Kar, and J. BlandHawthorn, "Ultrafast laser inscription of an integrated photonic lantern," Opt. Express 19, 5698-5705, March 2011.

[13] F. Hamaoka, S. Okamoto, K. Horikoshi, K. Yonenaga, A. Hirano and Y. Miyamoto, "Mode-selective coherent detection technique for lowcomplexity mode division multiplexing systems," Electronics Letters, vol. 51, no. 23, pp. 1899-1900, November 2015.

[14] F. Hamaoka, S. Okamoto, K. Horikoshi, k. Yonenaga, A. Hirano and Y. Miyamoto, "Mode and Polarization Division Multiplexed Signal Detection with Single Coherent Receiver Using Mode-Selective Coherent Detection Technique," Optical Fiber Communication Conference (OFC), Anaheim (California), paper Th3A.6, March 2016.

[15] H. Wen, C. Xia, A.M. Velzquez-Bentez, N. Chand, J.E. Antonio-Lopez, B. Huang, H. Liu, H. Zheng, P. Sillard, X. Liu, F. Effenberger, R. AmezcuaCorrea, and $\mathrm{G}_{i} \mathrm{Li}$, "First Demonstration of Six-Mode PON Achieving a Record Gain of $4 \mathrm{~dB}$ in Upstream Transmission Loss Budget," J. Lightwave Technol, vol. 34, no. 8, pp. 1990-1996, April 2016.

[16] J. B. Jensen, R. Rodes, A. Caballero, N. Cheng, D. Zibar and I. T. Monroy, "VCSEL Based Coherent PONs," J. Lightwave Technol, vol. 32, no. 8, pp. 1423-1433, April, 2014.

[17] J. D. Reis, D. M. Neves and A. L. Teixeira, "Analysis of Nonlinearities on Coherent Ultradense WDM-PONs Using Volterra Series," J. Lightwave Technol, vol. 30, no. 2, pp. 234-241, January 2012.

[18] V. Kamchevska, A.K. Medhin, F. Da Ros, F. Ye, R. Asif, A. Manolova Fagertun, S. Ruepp, M. Berger, L. Dittmann, T. Morioka, L. Oxenlwe, and M. Galili, "Experimental Demonstration of Multidimensional Switching Nodes for All-Optical Data Center Networks," J. Lightwave Technol. 34, 1837-1843, January 2016. 


\section{List of Figures}

1 Schematic and complexity comparison of: (a) conventional mode division multiplexed coherent transmission and (b) mode division multiplexed transmission based on frequency division multiplexed

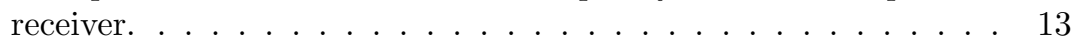

$2 \quad$ Experimental setup for $2.5 \mathrm{Gbit} / \mathrm{s} /$ mode (aggregate $10 \mathrm{Gbit} / \mathrm{s}$ ) transmission over $2.8 \mathrm{~km}$ 4-LP mode graded index fiber with mode frequency division multiplexed based coherent detection scheme. . . . . . . . . . . . . . . . . . . 14

3 Performance comparison of $2.5 \mathrm{Gbit} / \mathrm{s} /$ mode (aggregate $10 \mathrm{Gbit} / \mathrm{s}$ ) Nyquist QPSK transmission over $2.8 \mathrm{~km}$ 4-LP mode graded index fiber with frequency division multiplexed based coherent detection scheme. . . . . . . . . . . . . . . . . . 15

4 Receiver sensitivity at FEC threshold w.r.t different source wavelengths $(\lambda)$ for 4 -LP modes. $\ldots \ldots \ldots \ldots \ldots$

5 Simulated system performance (a) at various transmission distances of 4-LP mode graded index fiber and (b) number of LPmodes w.r.t the receiver bandwidth. $\ldots \ldots \ldots \ldots 17$ 


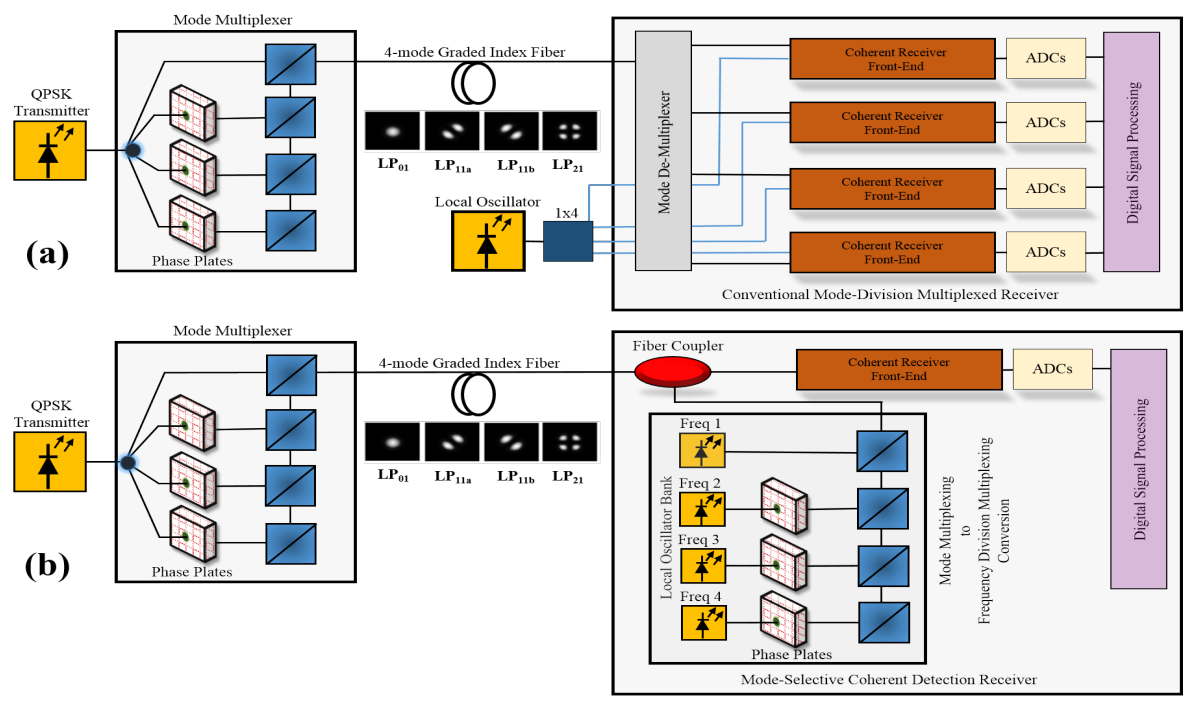

Figure 1: Schematic and complexity comparison of: (a) conventional mode division multiplexed coherent transmission and (b) mode division multiplexed transmission based on frequency division multiplexed receiver. 


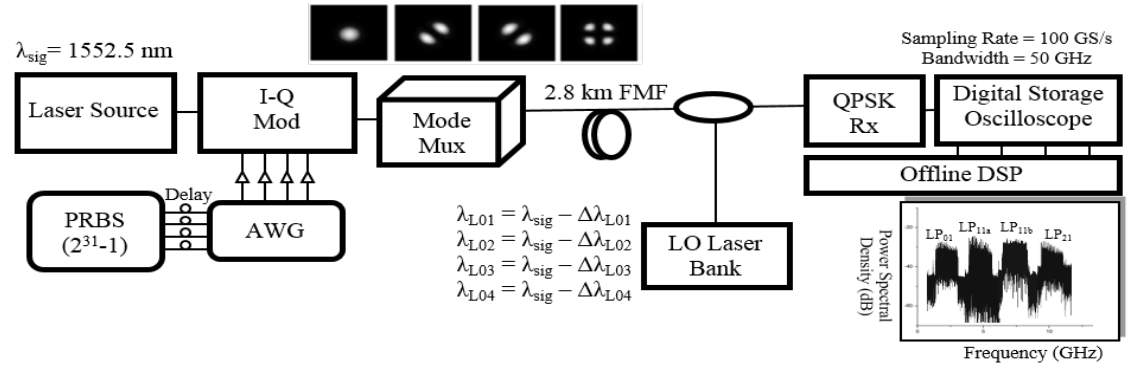

Figure 2: Experimental setup for $2.5 \mathrm{Gbit} / \mathrm{s} /$ mode (aggregate $10 \mathrm{Gbit} / \mathrm{s}$ ) transmission over 2.8 $\mathrm{km}$ 4-LP mode graded index fiber with mode frequency division multiplexed based coherent detection scheme. 


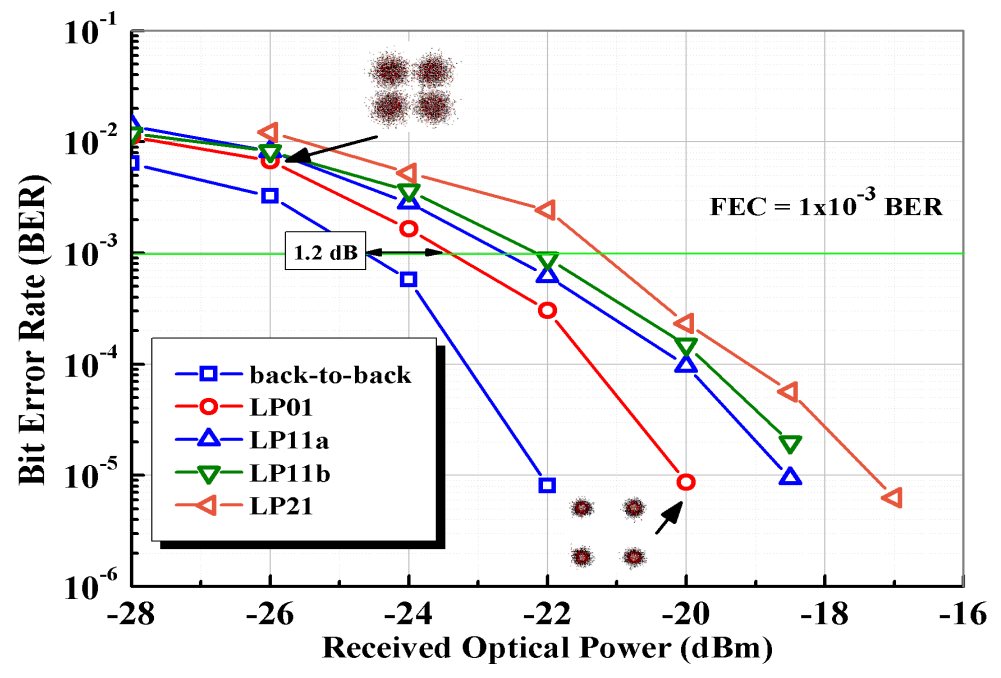

Figure 3: Performance comparison of $2.5 \mathrm{Gbit} / \mathrm{s} /$ mode (aggregate $10 \mathrm{Gbit} / \mathrm{s}$ ) Nyquist QPSK transmission over $2.8 \mathrm{~km}$ 4-LP mode graded index fiber with frequency division multiplexed based coherent detection scheme. 


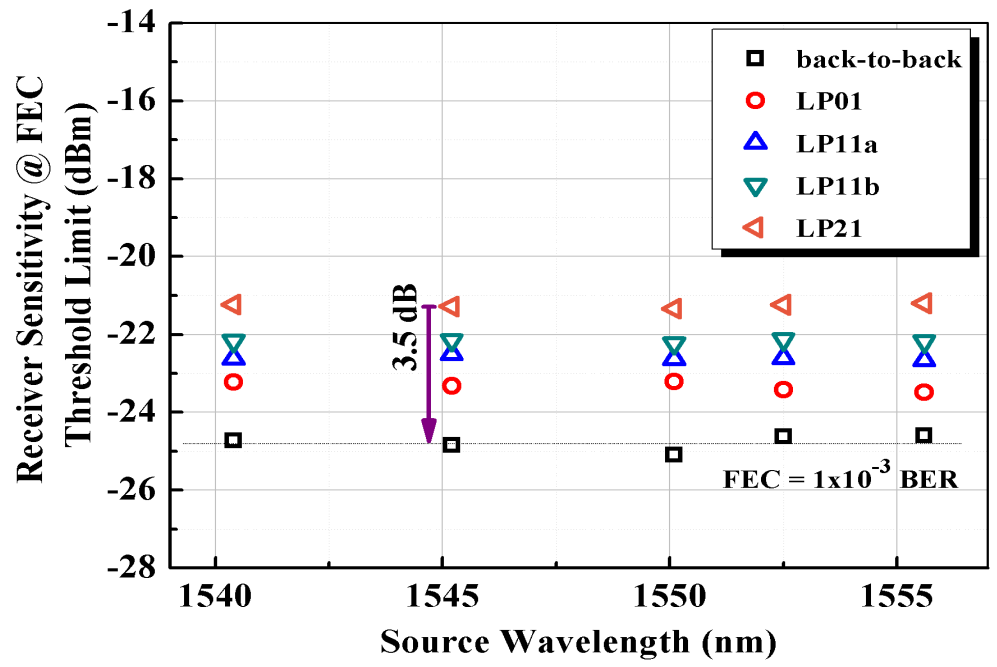

Figure 4: Receiver sensitivity at FEC threshold w.r.t different source wavelengths $(\lambda)$ for 4-LP modes. 

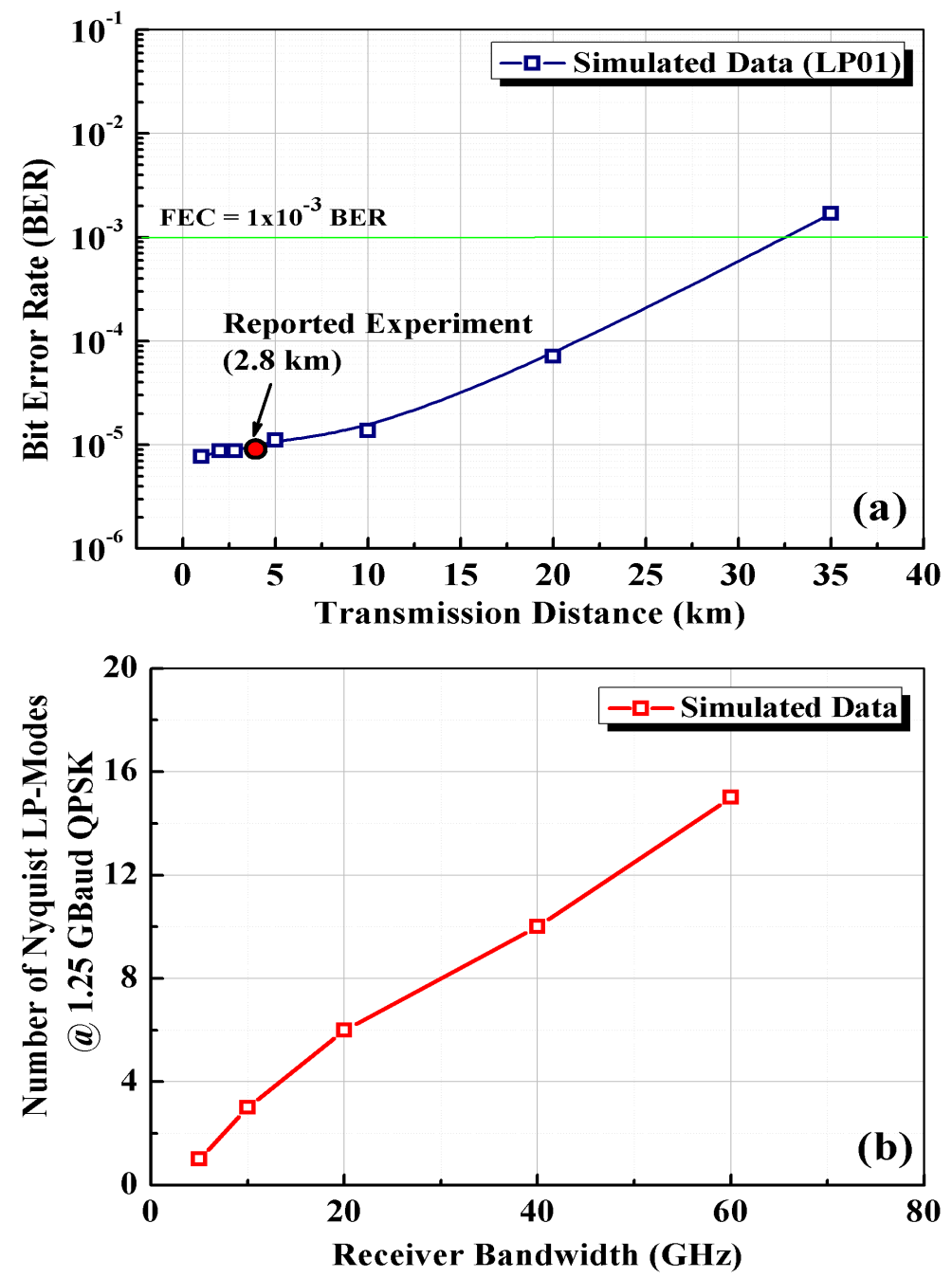

Figure 5: Simulated system performance (a) at various transmission distances of 4-LP mode graded index fiber and (b) number of LP-modes w.r.t the receiver bandwidth. 